\title{
Fast Prediction of Residual Stresses and Part Distortion in Selective Laser Melting
}

\author{
Tsung-Wen Tsai ${ }^{1}$, Tzong-Ming Wu ${ }^{1}$, Kuo-Teng Tsai ${ }^{1}$, Sung-Yueh Wu ${ }^{1}$, Sung-Ho Liu ${ }^{1}$, Ji-Bin \\ Horng ${ }^{1}$, Jinn-Kuen Chen ${ }^{2}$ \\ ${ }^{1}$ ITRI Southern Region Campus, Industrial Technology Research Institute \\ No. 8, Gongyan Rd., Liujia District, Tainan County 734, Taiwan \\ ${ }^{2}$ Department of Mechanical and Aerospace Engineering, University of Missouri \\ Columbia, MO 65211, U.S.A.
}

\section{Extended Abstract}

A 2D finite element model of multiphase fields was developed to investigate selective laser melting (SLM) of metal powders during additive manufacturing. The entire SLM process was simulated based on the three conservation equations of mass, momentum and energy. Multiple dynamic phenomena considered in this model include phase changes of solid/liquid and liquid/vapor, vapor pressure, surface tension, gravity, motion of molten liquid, and wetting of powders with liquid. To deposit laser energy to the mobile surfaces of powders, the liquid/vapor interface was tracked using a levelset method. All the physics were coupled, incorporated into and solved simultaneously in COMSOL Multyphysics ${ }^{\circledR}$. For comparison, an SLM experiment was performed for Ti6Al4V powders.

In the numerical analysis, the parameters of material and laser beams are the same as those used in the experiment. The average diameter (about $30 \mu \mathrm{m}$ ) of the Ti6Al4V powders is assumed for all the powder particles modeled. The power of the laser beam is $175 \mathrm{~W}$, and the laser spot radius at e-2 is $35 \mu \mathrm{m}$. Three laser scanning speeds considered are 1,650 $\mathrm{mm} / \mathrm{s}, 1,250 \mathrm{~mm} / \mathrm{s}$ and $850 \mathrm{~mm} / \mathrm{s}$. For simplicity, two computational models are used. The first model (I) consists of three powders in the direction perpendicular to the laser scanning direction, and the other model (II) consists of seven powders along the laser scanning direction. The latter is to simulate the height of the re-solidified layer, while the former is to simulate the cross-section profile produced by a single line scanning.

For the model I, the simulation results include temporal evolutions of the temperature distribution, flow velocity, and geometry profile of the melt pool. At the highest laser scanning speed $(1,650 \mathrm{~mm} / \mathrm{s})$ studied here, the powders partially melt, especially the two side ones. In this case of high scanning speeds, an AM part could be fabricated, but its shape and quality strongly depends on the scanning speed. As a result of more laser energy deposited into the powders by reducing the scanning speed, on the other hand, the central powder could significantly melt from the top surface and quickly lose its spherical shape due to the surface tension and gravity that immediately pull the newly liquefied material into the melt flow. After that part of molten liquid of the central powder touches the boundary of the side powders, formation of the melt pool begins and its dynamics can be manifested by the flow velocity field of the molten liquid. The powders could fully melt, consolidate and finally form a semi-circular cylinder. The simulated height and width of the re-solidified part are about $31.5 \mu \mathrm{m}$ and $83 \mu \mathrm{m}$ for the scanning speed $1,250 \mathrm{~mm} / \mathrm{s}$ and about $28 \mu \mathrm{m}$ and $104 \mu \mathrm{m}$ for the scanning speed $850 \mathrm{~mm} / \mathrm{s}$. The experimentally measurements are about $35.5 \mu \mathrm{m}$ and $113.5 \mu \mathrm{m}$ and about $26.8 \mu \mathrm{m}$ and $131 \mu \mathrm{m}$, respectively. It should be noted that no porosity in the consolidated part is found in the slowest scanning speed case ( $850 \mathrm{~mm} / \mathrm{s})$. For the model II, the average height of the simulated re-solidified track is about $\sim 20 \mu \mathrm{m}$, which is in a good correlation with the experimental observation that the compact layer has a realistic height of around $67 \%$ of the powder layer. 